\title{
Can the power Maxwell nonlinear electrodynamics theory remove the singularity of electric field of point-like charges at their locations?
}

\author{
B. Eslam Panah ${ }^{1,2,3 *}$ \\ 1 Department of Theoretical Physics, Faculty of Basic Sciences, \\ University of Mazandaran, P. O. Box 47416-95447, Babolsar, Iran \\ 2 ICRANet-Mazandaran, University of Mazandaran, P. O. Box 47416-95447, Babolsar, Iran \\ 3 ICRANet, Piazza della Repubblica 10, I-65122 Pescara, Italy
}

\begin{abstract}
YES! We introduce a variable power Maxwell nonlinear electrodynamics theory which can remove the singularity of electric field of point-like charges at their locations. One of the main problems of Maxwell's electromagnetic field theory is related to the existence of singularity for electric field of point-like charges at their locations. In other words, the electric field of a point-like charge diverges at the charge location which leads to an infinite self-energy. In order to remove this singularity a few nonlinear electrodynamics (NED) theories have been introduced. Born-Infeld (BI) NED theory is one of the most famous of them. However the power Maxwell (PM) NED cannot remove this singularity. In this paper, we show that the PM NED theory can remove this singularity, when the power of PM NED is less than $s<\frac{1}{2}$.
\end{abstract}

\section{INTRODUCTION}

The nonlinear field theories are interesting issues in physics because most of physical systems have a nonlinear behavior. As an example, the phenomenon of vacuum polarization in quantum electrodynamics, arising from the polarization of virtual electron-positron pairs and leading to nonlinear interactions between electromagnetic fields (an example that illustrates this is the scattering of photons by photons). The nonlinear electrodynamics (NED) theories come from the fact that these theories are generalizations of the linear Maxwell's theory and can remove some of problems Maxwell's theory and also in the special case they reduce to the Maxwell's theory. Other motivations of considering NED theories are limitations of the Maxwell theory 1, 2], description of the self-interaction of virtual electron-positron pairs 3 [5], the radiation propagation inside specific materials [6 - 9]. Also NED field modifies spacetime geometry (the gravitational red-shift) around superstrong magnetized compact objects [10 12], remove both of the big bang and black hole singularities 13 -17]. Moreover, the effects of NED field are important for studying higher magnetized neutron stars and pulsars [18, 19].

From a classical point of view, the existence of singularity at a point-like charge position is one of the main problems of Maxwell's electromagnetic field theory. In order to obtain a finite self-energy of a point charge, Born and Infeld (BI) have introduced a NED field by modifying Maxwell's theory at the short distance [20]. Another interesting properties of the BI theory is that its effective action arises in an open superstring theory and D-brains with non-singular selfenergy of the point-like charge [21 24]. Moreover, other NED theories were proposed in references [25 32], where this singularity is absent.

One of the interesting classes of the NED sources is power Maxwell (PM) NED theory. The Lagrangian this theory is an arbitrary power of Maxwell's Lagrangian [33 38], which is invariant under the conformal transformation $g_{\mu \nu} \rightarrow \Omega^{2} g_{\mu \nu}$, and $A_{\mu} \rightarrow A_{\mu}$ ( $g_{\mu \nu}$ and $A_{\mu}$ are metric tensor and electrical gauge potential, respectively). The PM NED theory is richer than linear Maxwell's theory and in a special case (unit power), reduces to linear Maxwell's theory [33 38]. In is notable that the coupling of PM NED theory with gravity always is an interesting subject which attracted significant attentions because of specific properties [39 50]. Another attractive feature of the PM NED theory is related to its conformal invariant. Indeed by adjusting the power of PM NED theory equals with a quarter of spacetime dimensions $(s=D / 4$ where $D$ and $s$ are dimensions of spacetime and power of PM NED theory, respectively), the theory is conformal invariant. In other words, for the special choice of $s=D / 4$, one obtains traceless energy-momentum tensor which leads to conformal invariant. It is notable that the idea is to take advantages of the conformal symmetry to construct the analogues of four-dimensional Reissner-Nordström solution with an inverse square electric field in arbitrary dimensions $\left(E \propto \frac{1}{r^{2}}\right.$, where $E$ is the electric field) [33, 51 53]. But the PM NED theory suffers from a main problem similar to linear Maxwell's theory and it is related to the existence of singularity for electromagnetic field of point-like charges at their locations. In order to remove this problem we study the behavior of PM NED theory with large value of $s$ at position of a point-like charge or near small charged

* email address: eslampanah@umz.ac.ir 
black hole. In other words, our aim is related to remove singularities electromagnetic field of point-like charges at their locations of the PM NED theory by considering a variable PM NED theory.

\section{THE ELECTRIC FIELD IN PM NED THEORY}

The 4-dimensional action of a NED is given by

$$
\mathcal{S}=\int d^{4} x \sqrt{-g} \mathcal{L}_{N E D}(\mathcal{F})
$$

where $\mathcal{L}_{N E D}(\mathcal{F})$ is an arbitrary Lagrangian of NED and $g:=\operatorname{det}\left(g_{\mu \nu}\right)$. Varying the action (1) with respect to the gauge potential $A_{\mu}$, we obtain the field equation as

$$
\partial_{\mu}\left(\sqrt{-g} \mathcal{L}_{F} F^{\mu \nu}\right)=0
$$

where $\mathcal{L}_{F}=d \mathcal{L}_{N E D}(\mathcal{F}) / d \mathcal{F}$, and $\mathcal{F}=F_{\mu \nu} F^{\mu \nu}$ is the Maxwell invariant. Also $F_{\mu \nu}=\partial_{\mu} A_{\nu}-\partial_{\nu} A_{\mu}$ is the electromagnetic tensor field.

It is well-known that the electric field is associated with the time component of the vector potential $\left(A_{t}\right)$. So we assume the vector potential as $A_{\mu}=h(r) \delta_{\mu}^{0}$.

The Lagrangian of PM NED theory is $\mathcal{L}_{N E D}(\mathcal{F})=(-\mathcal{F})^{s}$. By replacing the Lagrangian of PM NED theory in Eq. (21), we have

$$
\partial_{\mu}\left(\sqrt{-g}(-\mathcal{F})^{s-1} F^{\mu \nu}\right)=0
$$

Using the electrical gauge potential $\left(A_{\mu}=h(r) \delta_{\mu}^{0}\right)$ and Eq. (3), and by considering a 4-dimensional static spherical symmetric spacetime as $d s^{2}=-d t^{2}+d r^{2}+r^{2}\left(d \theta^{2}+\sin ^{2} \theta d \varphi^{2}\right)$, we obtain

$$
r h(r)^{\prime \prime}(2 s-1)+2 h(r)^{\prime}=0
$$

where the prime and the double prime denote the first and second derivatives with respect to $r$. Using the above equation we can extract the electrical gauge potential in the following form

$$
h(r)=q r^{\frac{2 s-3}{2 s-1}}
$$

where $q$ is an integration constant which is related to the electric charge.

Now we investigate the physical limit of power of the PM NED theory by evaluating the electrical gauge potential both at far from of the source $(r \rightarrow \infty)$ and at origin or at very short distance of the source $(r \rightarrow 0)$.

For $r \rightarrow \infty$ : the electrical gauge potential should be finite. Therefore, one should impose a restriction on $s$ as $\frac{2 s-3}{2 s-1}<0$, which leads to a restriction in the following form $\frac{1}{2}<s<\frac{3}{2}$.

For $r \rightarrow 0$ : by considering $s<\frac{1}{2}$, we can remove the singularity of electrical gauge potential of a point charge at its location. In other words, for $s<\frac{1}{2}, h(r)=q r^{\varepsilon}$ ( $\varepsilon$ is positive value), which indicate that the electrical gauge potential is finite when $r \rightarrow 0$.

So the physical limit of $s$ for the electrical gauge potential is as

$$
\text { Physical limit for } h(r) \text { leads to } \rightarrow\left\{\begin{array}{cc}
\frac{1}{2}<s<\frac{3}{2} \text { for } r \rightarrow \infty \\
s<\frac{1}{2} \quad \text { for } r \rightarrow 0
\end{array} .\right.
$$

Using Eq. (5), one can extract the electric field of PM NED theory as

$$
E(r)=\frac{d h(r)}{d r}=q r^{\frac{-2}{2 s-1}}
$$

We can remove the singularity of electrical field of a point charge at its location, when that $s$ is less than $\frac{1}{2}\left(s<\frac{1}{2}\right)$. In this case, the electrical field of PM NED theory turns to

$$
E(r)=q r^{\varepsilon}
$$



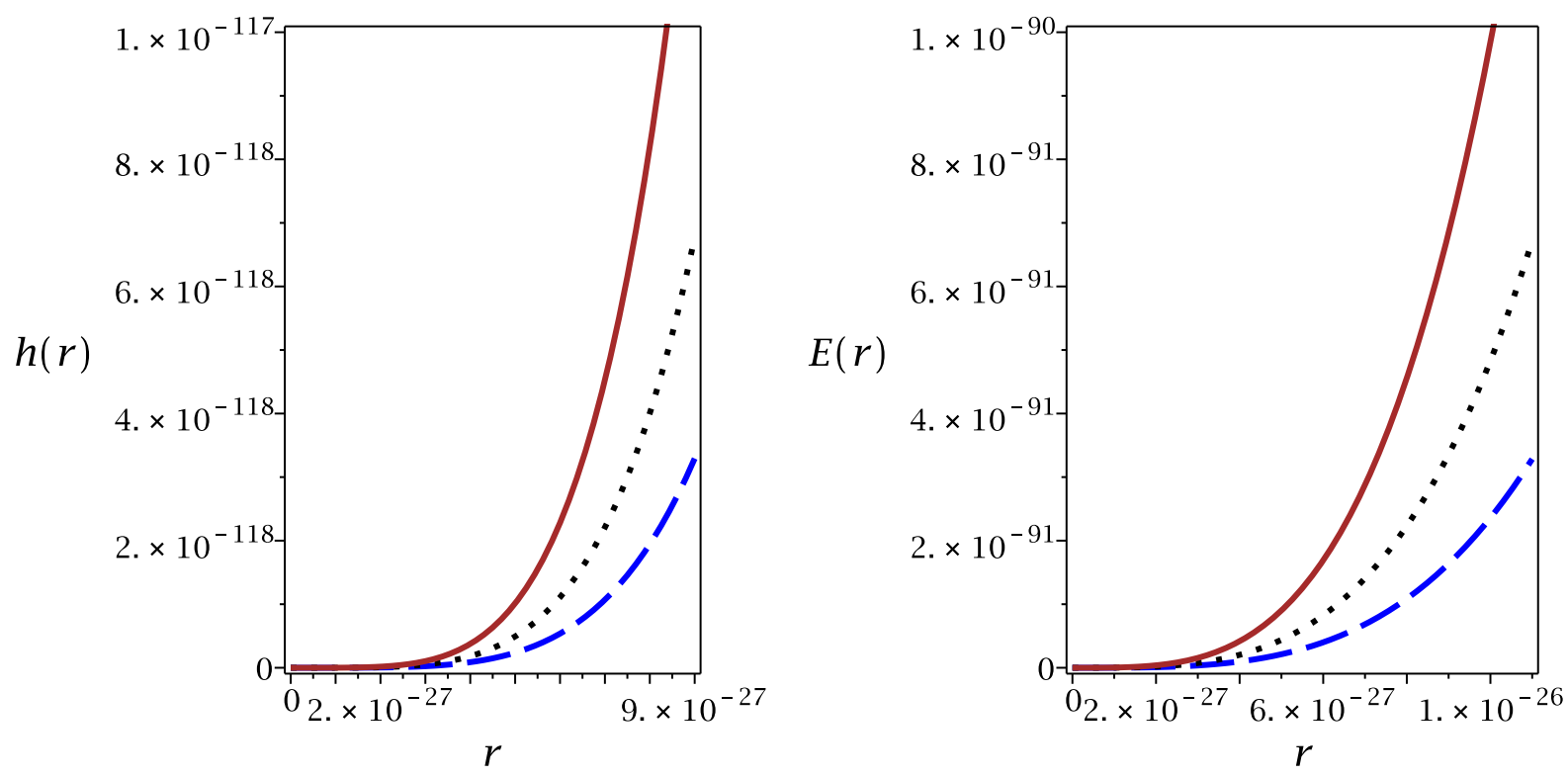

FIG. 1: $h(r)$ (left panel) and $E(r)$ (right panel) of point-like charges at near their locations versus $r$, for $q=0.1$, $s=0.212$ (dashed line), $s=0.211$ (dotted line) and $s=0.210$ (continuous line).

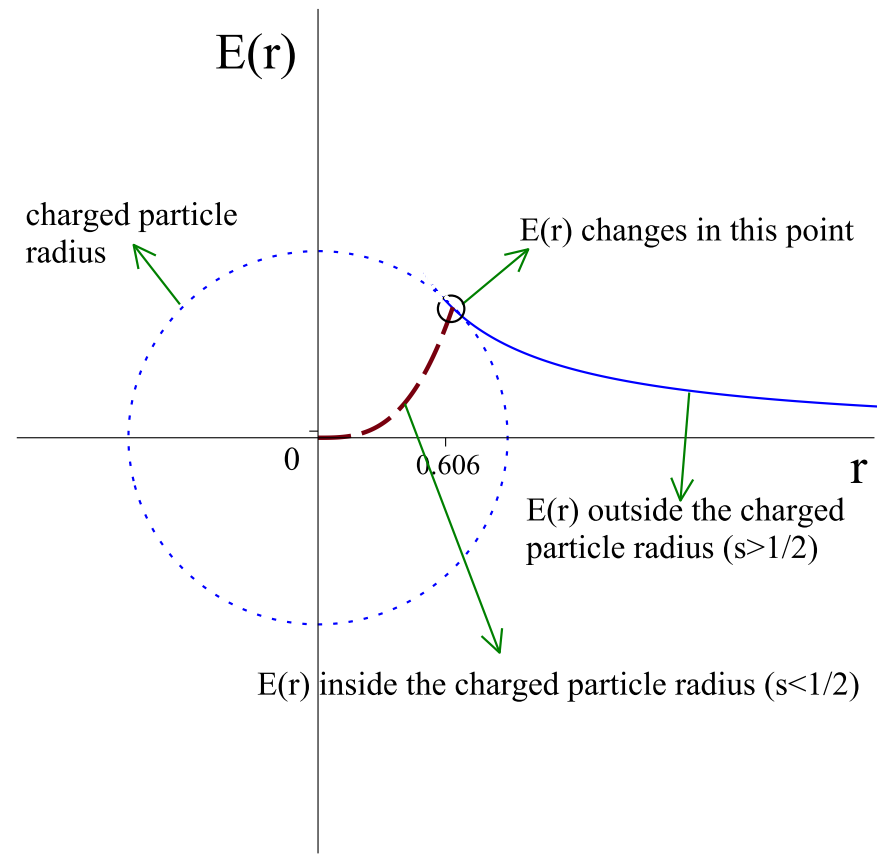

FIG. 2: $E(r)$ versus $r$, inside $(s<1 / 2)$ and outside $(s>1 / 2)$ of the charged particle radius.

where indicates the electrical field of point-charge in PM NED theory is a decreasing function of $r$ (for more details see Figs. 1 and 2).

On the other hand, the electrical charge should be zero at infinity distance of source $(r \rightarrow \infty)$. This fact impose a restriction on the range of $s$ as $s>\frac{1}{2}$. Therefore, we found the physical limit of $s$ for the electrical field as

Physical limit for $E(r)$ leads to $\rightarrow\left\{\begin{array}{l}s>\frac{1}{2} \text { for } r \rightarrow \infty \\ s<\frac{1}{2} \text { for } r \rightarrow 0\end{array}\right.$. 
TABLE I: Acceptable ranges of $s$ based on the physical limit of the electrical gauge potential and the electric field.

\begin{tabular}{||c|c|c||c|c||}
\hline \hline different fields & $h(r)$ when $r \rightarrow \infty$ & $h(r)$ when $r \rightarrow 0$ & $E(r)$ when $r \rightarrow \infty$ & $E(r)$ when $r \rightarrow 0$ \\
\hline \hline acceptable ranges of $s$ & $\frac{1}{2}<s<\frac{3}{2}$ & $s<\frac{1}{2}$ & $s>\frac{1}{2}$ & $s<\frac{1}{2}$ \\
\hline \hline
\end{tabular}

See Table. 【 for more details about acceptable ramges of $s$ based on the physical limit of the electrical gauge potential and the electric field.

So the PM NED theory is a theory without any singularities of the electric field of point-like charges at their locations when $s$ is less than $\frac{1}{2}\left(s<\frac{1}{2}\right)$.

In this paper we indicated that the PM NED theory can avoid of singularities of the electrical gauge potential and the electric field of point-like charges at their locations, when $s<\frac{1}{2}$.

\section{CONCLUSION}

In this paper, we have introduced a variable PM NED theory which can remove the singularity of electric field of point-like charges at their locations, when $s<\frac{1}{2}$. Indeed, we have indicated that the electrical field of a point-like charge in the variable PM NED theory is finite at $r \rightarrow 0$. Also, we have obtained acceptable ranges of power of PM NED $(s)$ for all spacetime in Table. I]

[1] D. H. Delphenich, Nonlinear electrodynamics and QED. arXiv:hep-th/0309108.

[2] D. H. Delphenich, Nonlinear optical analogies in quantum electrodynamics. arXiv:hep-th/0610088.

[3] W. Heisenberg and H. Euler, Z. Phys. 98 (1936) 714 arXiv:physics/0605038 (Translation by: W. Korolevski and H. Kleinert, Consequences of Dirac's Theory of the Positron)].

[4] J. Schwinger, Phys. Rev. 82 (1951) 664.

[5] H. Yajima, and T. Tamaki, Phys. Rev. D 63 (2001) 064007.

[6] V. A. De Lorenci, and M. A. Souza, Phys. Lett. B 512 (2001) 417.

[7] V. A. De Lorenci, and R. Klippert, Phys. Rev. D 65 (2002) 064027.

[8] M. Novello et al., Class. Quant. Gravit. 20 (2003) 859.

[9] M. Novello, and E. Bittencourt, Phys. Rev. D 86 (2012) 124024.

[10] A. Ibrahim, et al., Astrophys. J. Lett. 574 (2002) L51.

[11] A. Ibrahim, et al., Astrophys. J. Lett. 584 (2003) L17.

[12] H. J. Mosquera Cuesta, and J. M. Salim, Mon. Not. Roy. Astron. Soc. 354 (2004) L55.

[13] E. Ayon-Beato, and A. Garcia, Gen. Relat. Gravit. 31 (1999) 629.

[14] E. Ayon-Beato, and A. Garcia, Phys. Lett. B 464 (1999) 25.

[15] V. A. De Lorenci, R. Klippert, M. Novello, and J.M. Salim, Phys. Rev. D 65 (2002) 063501.

[16] I. Dymnikova, Class. Quant. Gravit. 21 (2004) 4417.

[17] C. Corda, and H. J. Mosquera Cuesta, Mod. Phys. Lett A 25 (2010) 2423.

[18] Z. Bialynicka-Birula, and I. Bialynicka-Birula, Phys. Rev. D 2 (1970) 2341.

[19] H. J. Mosquera, and J. M. Cuesta Salim, Astrophys. J. 608 (2004) 925.

[20] M. Born, and L. Infeld, Proc. Roy. Soc. Lond. 144 (1934) 425.

[21] E. S. Fradkin, and A. A. Tseylin, Phys. Lett. B 163 (1985) 123.

[22] D. L. Wiltshire, Phys. Rev. D 38 (1988) 2445.

[23] R. G. Leigh, Mod. Phys. Lett. A 4 (1989) 2767.

[24] G. W. Gibbons, and C. A. R. Herdeiro, Class. Quant. Grav. 18 (2001) 1677.

[25] I. Dymnikova, Gen. Rev. Grav. 24 (1992) 235.

[26] H. H. Soleng, Phys. Rev. D 52 (1995) 6178.

[27] I. Dymnikova, Class. Quant. Grav. 21 (2004) 4417.

[28] I. Dymnikova, and E. Galaktionov, Class. Quant. Grav. 32 (2015) 165015.

[29] D. M. Gitman, and A. E. Shabad, Eur. Phys. J. C 74 (2014) 3186.

[30] S. I. Kruglov, Ann. Phys. (Berlin) 527 (2015) 397.

[31] S. I. Kruglov, Eur. Phys. J. C 75 (2015) 88.

[32] S. I. Kruglov, Commun. Theor. Phys. 66 (2016) 59.

[33] M. Hassaine, and C. Martinez, Phys. Rev. D 75 (2007) 027502.

[34] M. Hassaine, and C. Martinez, Class. Quantum Gravit. 25 (2008) 195023. 
[35] H. Maeda, M. Hassaine, and C. Martinez, Phys. Rev. D 79 (2009) 044012.

[36] S. H. Hendi, Phys. Lett. B 678 (2009) 438.

[37] S. H. Hendi, Class. Quantum Gravit. 26 (2009) 225014.

[38] S. H. Hendi, B. Eslam Panah, and R. Saffari, Int. J. Mod. Phys. D 23 (2014) 1450088.

[39] S. H. Hendi, Phys. Lett. B 690 (2010) 220.

[40] S. H. Hendi, and B. Eslam Panah, Phys. Lett. B 684 (2010) 77.

[41] O. Gurtug, S. H. Mazharimousavi, and M. Halilsoy, Phys. Rev. D 85 (2012) 104004.

[42] M. Kord Zangeneh, M. H. Dehghani, and A. Sheykhi, Phys. Rev. D 92 (2015) 104035.

[43] J. -X. Mo, G. -Q. Li, and X. -B. Xu, Phys. Rev. D 93 (2016) 084041.

[44] S. H. Hendi, B. Eslam Panah, S. Panahiyan, and M. Momennia, Eur. Phys. J. C 77 (2017) 647.

[45] W. Xu, and D. -C. Zou, Gen. Rel. Grav. 49 (2017) 73.

[46] H. -F. Li, X. -y. Guo, H. -H. Zhao, and R. Zhao, Gen. Rel. Grav. 49 (2017) 111.

[47] S. H. Hendi, B. Eslam Panah, S. Panahiyan, and M. S. Talezadeh, Eur. Phys. J. C 77 (2017) 133.

[48] A. Rincon, and G. Panotopoulos, Phys. Rev. D 97 (2018) 024027.

[49] S. H. Hendi, B. Eslam Panah, and S. Panahiyan, Fortschr. Phys. 66 (2018) 1800005.

[50] M. M. Stetsko, Phys. Rev. D 99 (2019) 044028.

[51] S. H. Hendi, and H. R. Rastegar-Sedehi, Gen. Relativ. Gravit. 41 (2009) 1355.

[52] S. H. Hendi, Phys. Lett. B 677 (2009) 123.

[53] D. Roychowdhury, Phys. Lett. B 718 (2013) 1089. 